\title{
An Inverse Problem Approach for the Estimation of the Haverkamp and van Genuchten Retention Curves Parameters with the Luus-Jaakola Method
}

\author{
F. F. FERREIRA ${ }^{1 *}$, M. DE O. TEMPERINI ${ }^{2}$, W. R. TELLES ${ }^{3}$, \\ G. B. LYRA ${ }^{4}$ and A. J. SILVA NETO 5
}

Received on January 15, 2020 / Accepted on January 18, 2021

\begin{abstract}
In academia, the study of the movement of water in the ground has been widespread since the last century. The same can be determined using the Richards equation. This is a nonlinear parabolic partial differential equation that requires parameters to generate the results of the problem. Some authors have proposed equations that represent the relationship between volumetric moisture and soil water potential, such as Haverkamp and van Genuchten. As main objective of this work, the inverse modeling was implemented to obtain the Richards equation parameters, applying the Luus-Jaakola method. To verify the mathematical model, a sensitivity analysis was performed, which allowed the observation of the effect that each parameter has on the output data, implying a linear dependence. The results produced proved to be satisfactory for the problem analyzed in our research.
\end{abstract}

Keywords: Richards equation, inverse problem, Luus-Jaakola.

\section{INTRODUCTION}

The movement of water and solutes (e.g. nutrients and pesticides) in the soil is an important process in studies of management and conservation of water resources. The spatiotemporal variability of groundwater movement allows the determination of variables such as water content,

\footnotetext{
*Corresponding author: Fabio Freitas Ferreira - E-mail: fabiofreitasferreira@id.uff.br

${ }^{1}$ Department of Natural Sciences, IHS, Universidade Federal Fluminense, Rua Recife s/n, 28895-514, Rio das Ostras, RJ, Brazil - E-mail: fabiofreitasferreira@id.uff.br https://orcid.org/0000-0001-5878-7078

${ }^{2}$ Graduate Program in Biosystems Engineering, Engineering School, UFF, Niterói, RJ, Brazil - E-mail: marcelotemperini @id.uff.br https://orcid.org/0000-0001-9498-903X

${ }^{3}$ Department of Exact, Biological and Earth Sciences, UFF, Santo Antônio de Pádua, RJ, Brazil - E-mail: wtelles@id.uff.br https://orcid.org/0000-0002-6032-3405

${ }^{4}$ Department of Environmental Sciences, Forest Institute, UFRRJ, Seropédica, RJ, Brazil - E-mail: gblyra@ufrrj.br https://orcid.org/0000-0002-9882-7000

${ }^{5}$ Department of Mechanical Engineering and Energy, Polytechnic Institute, UERJ, Nova Friburgo, RJ, Brazil - E-mail: ajsneto@ iprj.uerj.br https://orcid.org/0000-0002-9616-6093
} 
hydraulic conductivity, initial and boundary conditions, required in various applications in engineering, soil physics, meteorology, hydrology and groundwater. Stand out among these applications, hydrologic budget, irrigation and drainage, prediction of contaminant transport in the vadose zone, aquifer recharge, climate and meteorological modeling, soil-water-plant modeling and waste management $[9,20,22]$.

The water transport in unsaturated soil can be described numerically using Richards equation, which combines Darcy's law and the continuity equation. However, for its solution, it is necessary to determine the empirical nonlinear relationship between soil water content and pressure head, described by a water retention curve. The water retention curve can be represented by several empirical models (e.g. van Genutchen, Haverkamp and Campbell) $[3,8,19]$, in which their coefficients must betted to different soil types. The values of these coefficients available in the literature are not accurate and are often presented in intervals for soil texture classes [15] or obtained by pedotransfer functions. The determination of the coefficients using traditional methods (e.g. Richards pressure plate apparatus) demands a significant amount of time and financial resources. Thus, its determination by inverse problem methods is an alternative to obtain coefficients that best represent the infiltration for a given soil.

For the application of the inverse problem methods it is necessary to solve the direct problem, that is, Richards equation must be resolved. The Richards equation is a nonlinear parabolic partial differential equation whose solution is not trivial $[15,18]$. Several numerical methods are used for your solution, e.g. the Finite Difference Method [4], the Finite Volume Method [2] and the Finite Element Method [5, 6, 18]. However, the Finite Volumes Method result in stability for its solution, being easy to implement and, mainly, it is a conservative method, which allows to keep the properties of the equation [18].

The inverse problem methods can be divided into deterministic and stochastic [13]. Most deterministic methods require accurate experimental measurements of the problem, and often stop at a local minimum and thus do not return to the solution for the optimal global [15]. Sawadogo et al. [15] commit that deterministic algorithms still require regularity of functions to be optimized. However, this regularity is not often verified. Stochastic methods generate random variables through predefined functions, allowing other regions within the problem domain to be explored away from the minimum locations already found [17].

\section{MATHEMATICAL MODEL}

Consider the values obtained experimentally for the soil water content, $\boldsymbol{\theta}_{e}$. The residue between the calculated, $\boldsymbol{\theta}_{c}$, obtained with the solution of a mathematical/computational model, and the experimental quantity is given by

$$
\mathbf{R}=\boldsymbol{\theta}_{c}-\boldsymbol{\theta}_{e}
$$

where the subscript $c$ indicates the value of $\theta$ calculated computationally, and the subscript $e$ indicates the value of $\theta$ obtained experimentally in the field, $\theta\left(\mathrm{m}^{3} \mathrm{~m}^{-3}\right)$. The objective with the solution of the inverse problem for the determination of estimates for the unknown parameters 
of the model is that the residue be as small as possible. Then, there is a minimization problem to be tackled.

The functional of square residues is given by

$$
Q(\mathbf{P})=\frac{1}{2}|\mathbf{R}|^{2}=\frac{1}{2} \mathbf{R}^{T} \mathbf{R}
$$

where $\mathbf{R}=\left(R_{1}, \ldots, R_{M}\right)^{T} \in \mathbb{R}^{M}$ represents the vector of residues, $M$ is the amount of experimental data, and $\mathbf{P}$ represents the parameters to be estimated, or, in other words, the solution of the inverse problem [13].

Replacing (2.1) in (2.2), results

$$
Q(\mathbf{P})=\frac{1}{2} \sum_{i=1}^{M}\left(\theta_{c_{i}}-\theta_{e_{i}}\right)^{2}
$$

\subsection{Richards' One-Dimensional Equation}

In order to solve water infiltration problems in soil, the Richards equation, described in its $\psi$ based form, is given by [14]

$$
C(\psi) \frac{\partial \psi}{\partial t}=\frac{\partial}{\partial z}\left[K(\psi) \frac{\partial(\psi-z)}{\partial z}\right]
$$

where $\psi$ is the pressure head $(\mathrm{cm}), C(\psi)$ is the water capacity $\left(\mathrm{cm}^{-1}\right), t$ is the time $(s), K(\psi)$ is the hydraulic conductivity $\left(\mathrm{cm} \mathrm{s}^{-1}\right)$, and $z$ is the vertical coordinate $(\mathrm{cm})$, from the origin to the negative axis, $-z[5,17,18]$. The initial and the boundary conditions will be defined in section 5 .

The equation for modeling the retention curve has already been proposed by several authors [5]. In this work the curves of van Genuchten (eq. 2.5) [19] and of Haverkamp (eq. 2.6) [8] were chosen. They relate the soil water content and the pressure head, i.e., $\theta=\theta(\psi)$, and are given, respectively, by

$$
\begin{gathered}
\theta(\psi)=\theta_{r}+\left(\theta_{s}-\theta_{r}\right)\left(1+|\alpha \psi|^{n}\right)^{-m} \\
\theta(\psi)=\theta_{r}+\frac{B\left(\theta_{s}-\theta_{r}\right)}{B+|\psi|^{\lambda}}
\end{gathered}
$$

The van Genuchten (eq. 2.7) and Haverkamp (eq. 2.8) hydraulic conductivity equations are given by

$$
\begin{gathered}
K(\psi)=K_{s}\left(1-(\alpha|\psi|)^{n-1}\left[1+(\alpha|\psi|)^{n}\right]^{-m}\right)^{2}\left[1+(\alpha|\psi|)^{n}\right]^{-m / 2} \\
K(\psi)=K_{s} \cdot \frac{A}{A+|\psi|^{\phi}}
\end{gathered}
$$

In equations (2.5) to (2.8), we have that $\theta_{s}$ is the saturated water content $\left(\mathrm{cm}^{3} \mathrm{~cm}^{-3}\right), \theta_{r}$ is the residual water content after drying the soil $\left(\mathrm{cm}^{3} \mathrm{~cm}^{-3}\right), K_{s}$ is the saturated hydraulic conductivity $\left(\mathrm{cm} \mathrm{s}^{-1}\right)$, and $\alpha\left(\mathrm{cm}^{-1}\right), n$ and $m$ (dimensionless) are empirical factors. The parameters $m$ and $n$ are related by $m=1-1 / n,[11]$. In eqs. (2.6) and (2.8) $\lambda$ is an index related to the distribution of soil pores $(\mathrm{cm})$, and $A, B$ and $\phi$ are dimensionless parameters. 


\subsection{Direct Problem Solution}

In regard to solving flow problems in porous media, a fundamental characteristic sought is the observance of mass conservation [5], an essential requirement for the solution to have physical meaning. Then, since the conservation of the physical quantities at the discrete level is an intrinsic feature of the Finite Volume Method (FVM), this method was implemented to solve the Richards equation [18].

\section{SENSITIVITY ANALYSIS}

In a mathematical model, sensitivity analysis allows the observation of the effect that each parameter has on the output data. Thus, it can be verified if the mathematical model is sensitive to any parameter, and which parameters are possibly correlated, implying a linear dependence [7].

The scaled sensitivity coefficients are given by

$$
X_{i}=\mathbf{P}_{i} \cdot \frac{\partial \theta}{\partial \mathbf{P}_{i}} \quad i=1,2, \ldots, N
$$

where $N$ is the total of parameters to be determined.

Two distinct values of the same parameter are expected to return two distinct solutions to the direct problem, thus showing the sensitivity of the model to the parameter. Otherwise, the sensitivity coefficient is of small magnitude or even null, the parameter will not be accurately determined with the solution of the inverse problem.

The correlation between the parameters of the mathematical model can be analyzed by means of the sensitivity coefficient ratio, given by

$$
\bar{X}_{i j}=\frac{X_{i}}{X_{j}}, \quad i \neq j, \quad i, j=1,2, \ldots, N
$$

The sensitivity coefficient ratio between two parameters indicates whether they are linearly dependent or independent. Two parameters being correlated implies that they cannot be simultaneously obtained.

\subsection{Sensitivity to the van Genuchten Parameters}

This section presents the sensitivity analysis for the van Genuchten retention curve parameters. The parameters analyzed are $\alpha, n, \theta_{r}, \theta_{s}$ and $K_{s}$. The case used to perform the sensitivity analysis is presented in section 5.1. The sensitivity coefficient values for each parameter varies with the soil type. Therefore, these results cannot be generalized, but serve as the basis of a preliminary evaluation. The results are shown in Figure 1.

It is observed that all the sensitivity coefficients obtained are significant, that is, the mathematical model is sensitive to all parameters. The parameters sensitivity coefficients ratios can be seen in Figure 2. 


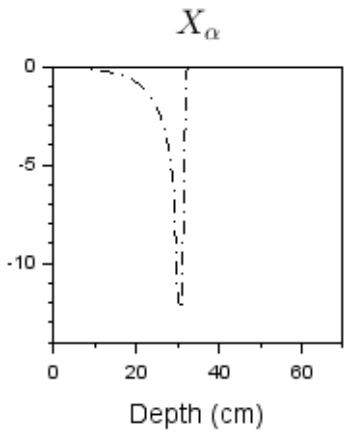

$X_{\theta_{s}}$

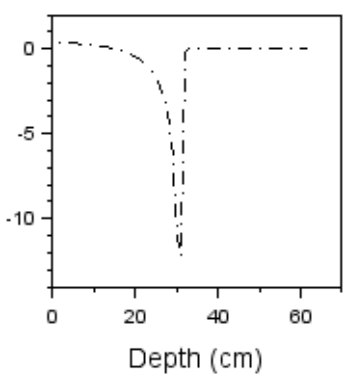

$X_{n}$

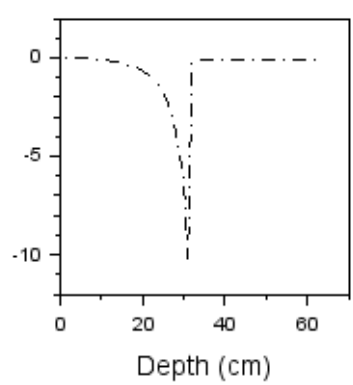

$X_{K_{s}}$

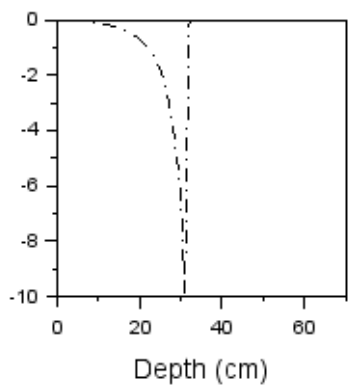

$X_{\theta_{r}}$

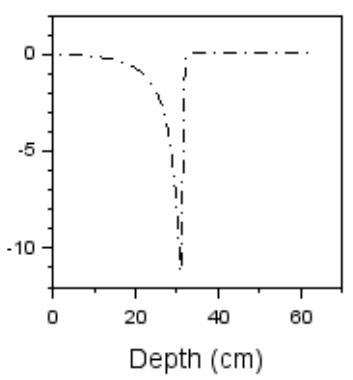

Depth $(\mathrm{cm})$

Figure 1: Sensitivity coefficients $X_{i}$ for the van Genuchten retention curve parameters.
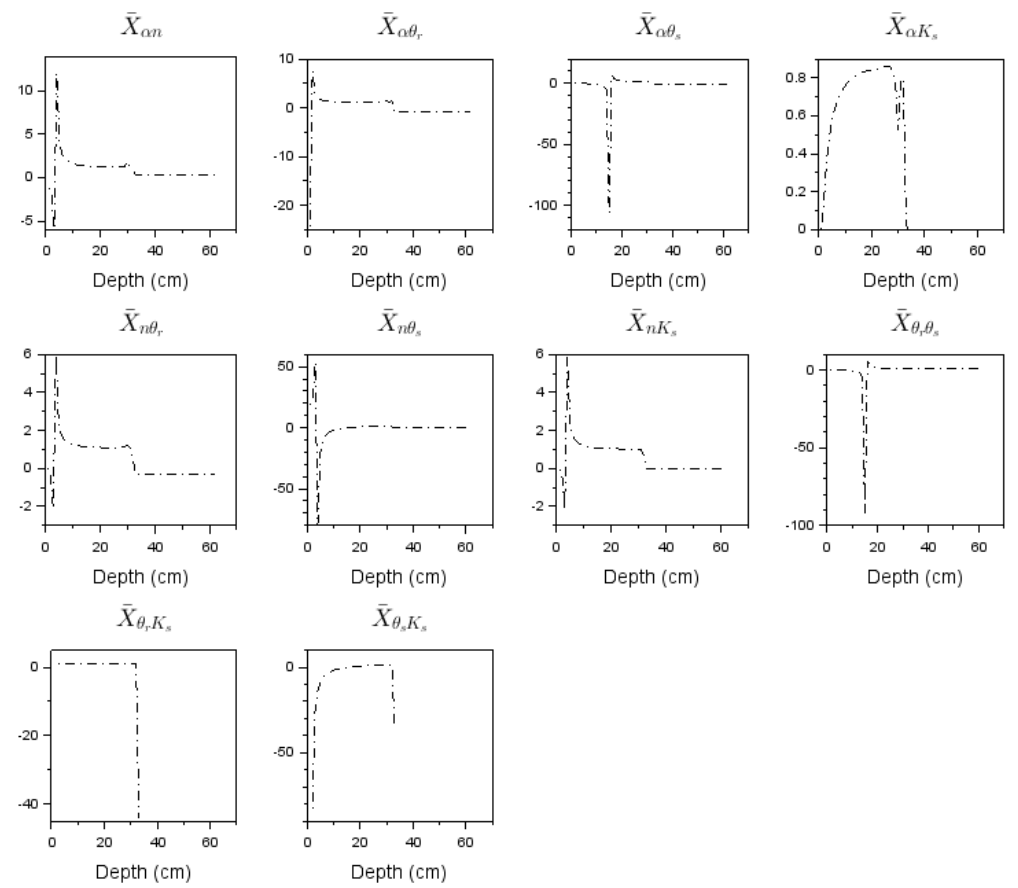

Figure 2: Sensitivity coefficients ratios, $\bar{X}_{i j}$, for the van Genuchten retention curve parameters. 
Although the correlation graph between $n$ and $\theta_{s}$ be almost constant throughout the depth, there is a slight variation between the depths from $0 \mathrm{~cm}$ to $10 \mathrm{~cm}$. Thus, neither of both parameters will be excluded from the search. For all other combinations, there are no linear correlations.

\subsection{Sensitivity to the Haverkamp Parameters}

This section presents the sensitivity analysis for the Haverkamp's retention curve parameters. The parameters analyzed are $A, \phi, B, \lambda, \theta_{r}, \theta_{s}$ and $K_{s}$. The case used to perform the sensitivity analysis is presented in section 5.2. The results are shown in Figure 3.

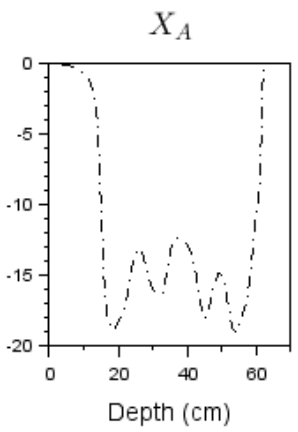

$X_{\theta_{r}}$

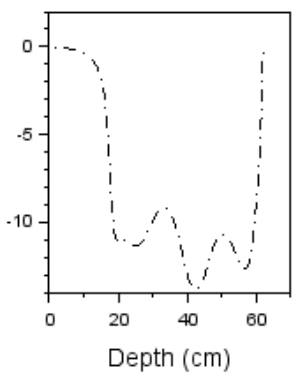

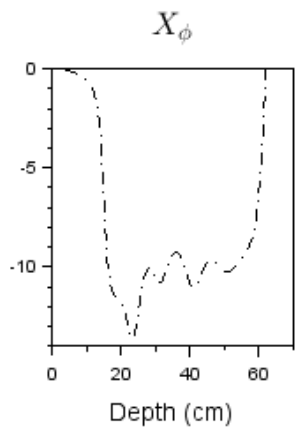

$X_{\theta s}$

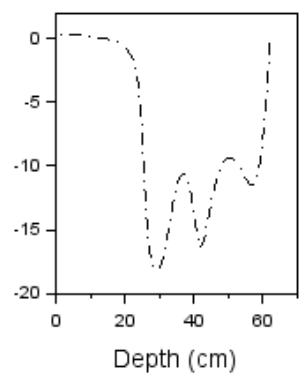

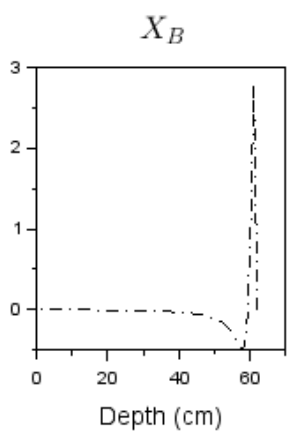

$X_{K s}$

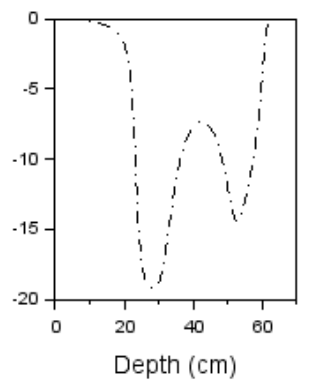

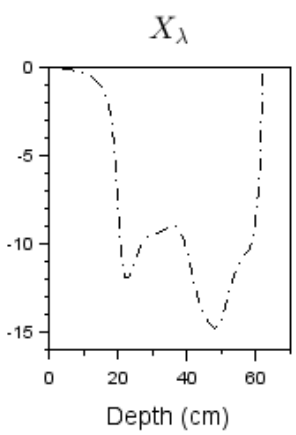

Depth $(\mathrm{cm})$

Figure 3: Sensitivity coefficients $X_{i}$ for the Haverkamp retention curve parameters.

It is observed that all parameters sensitivity coefficients are significant, concluding that the mathematical model is sensitive to each parameter. The next analysis is the sensitivity coefficients ratios, shown in Figure 4.

It is observed in Figure 4 that the graphs are not constant, although some coefficients show this behavior at certain depths. Some difficulty may be antecipated in the simulations estimations of some parameters, such as $B-\lambda, B-\theta_{r}, B-K_{s}, \lambda-\theta_{r}$ and $\theta_{s}-K_{s}$.

\section{INVERSE PROBLEM SOLUTION}

Luus and Jaakola $[12,16]$ developed a simple optimization procedure to solve nonlinear programming problems. The procedure applied to the solution of the inverse problem of the interest 

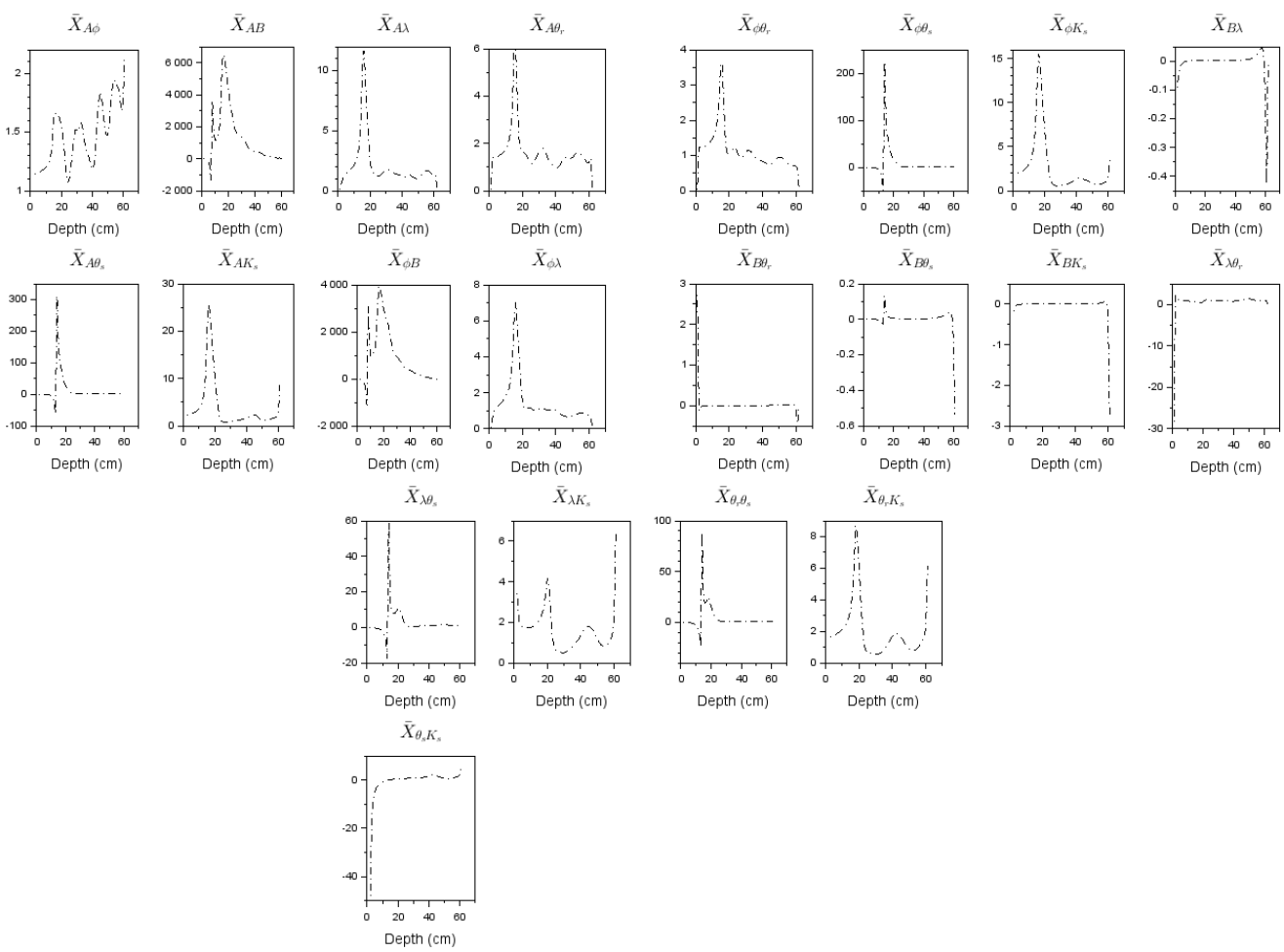

Figure 4: Sensitivity coefficients rates, $\bar{X}_{i j}$, for the Haverkamp retention curve parameters.

in the present work is based on minimizing the functional given by eq. (2.3). For that purpose, restrictions on the search domain for the parameters are defined as

$$
\operatorname{MIN}_{\mathbf{P}}<\mathbf{P}<M A X_{\mathbf{P}}
$$

where $M I N_{\mathbf{P}}$ and $M A X_{\mathbf{P}}$ are respectively the vectors containing the lower and upper limits of the search interval for the parameters of interest (vector $\mathbf{P}$ ), and \#(P) is the number of parameters to be estimated.

This is a minimization problem with restrictions. The Luus-Jaakola's method is described in the algorithm below.

1. Read the vector with experimental data $\boldsymbol{\theta}_{\boldsymbol{e}}$.

2. The restrictions are defined, i.e., the maximum value and the minimum value for $\mathbf{P}$, see eq. (4.1), according to the existing literature.

3. An initial random estimate (candidate solution) is generated, within the constraints described in the previous step. Denote these initial values as $\mathbf{P}^{0}$, and the amplitude of the search interval $\mathbf{r}^{0}=M A X_{\mathbf{P}^{0}}-\operatorname{MIN}_{\mathbf{P}^{0}}$; 
4. Solve Richard's equation, i.e. eq. (2.4), and calculate the residue $Q_{0}=Q\left(\mathbf{P}^{0}\right)$ according to eq. (2.3);

5. Define the number of times that the amplitude of the search interval will be reduced. Denote this amount by $N_{\text {out }}$.

6. Define a number of possible candidate solutions, $N_{\text {int }}$.

7. For $i=1: N_{\text {out }}$ do:

(a) For $j=1: N_{\text {int }}$ do:

i. Generate random numbers between -0.5 and +0.5 for each of the parameters to be determined. Denote these by $\mathbf{Y}$;

ii. Take random numbers from step (7ai) and attribute to the new candidate solution $\mathbf{P}, \mathbf{P}^{\text {new }}$, given by

$$
P_{k}^{i, j}=P_{k}^{i-1, j}+Y_{k} \cdot r_{k}^{i-1} \quad, \quad k=1, \ldots, \#(\mathbf{P})
$$

iii. Test the restrictions imposed for each $P_{k}, i=1, \ldots, \#(\mathbf{P})$. If $P_{k}>M A X_{P_{k}}$, then do $P_{k}=M A X_{P_{k}}$. Se $P_{k}<M I N_{P_{k}}$, then do $P_{k}=M I N_{P_{k}}$;

iv. Calculate the new value $\boldsymbol{\theta}_{c}$, using eq. (2.4);

v. Calculate the new residue $Q_{\text {new }}=Q\left(\mathbf{P}^{\text {new }}\right)$ according to eq. (2.3);

vi. If $Q_{\text {new }}<Q_{0}$, then assume the new parameters obtained at random as the optimal solution of the problem and make $Q_{0}=Q_{n e w}$. Otherwise, discard the new values for $\mathbf{P}$;

(b) If $i$ did not reach $N_{\text {out }}$, reduce the amplitude of the search interval by a unit fee pre-defined in the algorithm, called $\varepsilon, \mathbf{r}^{i}=(1-\varepsilon) \mathbf{r}^{i-1}$ with $0<\varepsilon<1$;

8. At the end of the procedure $\mathbf{P}$ is the best candidate solution which minimizes the functional $Q(\mathbf{P})$.

In Figure 5 the flowchart of the Luus-Jaakola method is presented.

\section{RESULTS AND DISCUSSION}

The numerical results obtained are presented next. As described previously, the direct problem was solved using the Finite Volume Method (FVM), and the Inverse Problem with the LuusJaakola method. Both algorithms were implemented using SciLab [1], a free software developed for scientific work research. 


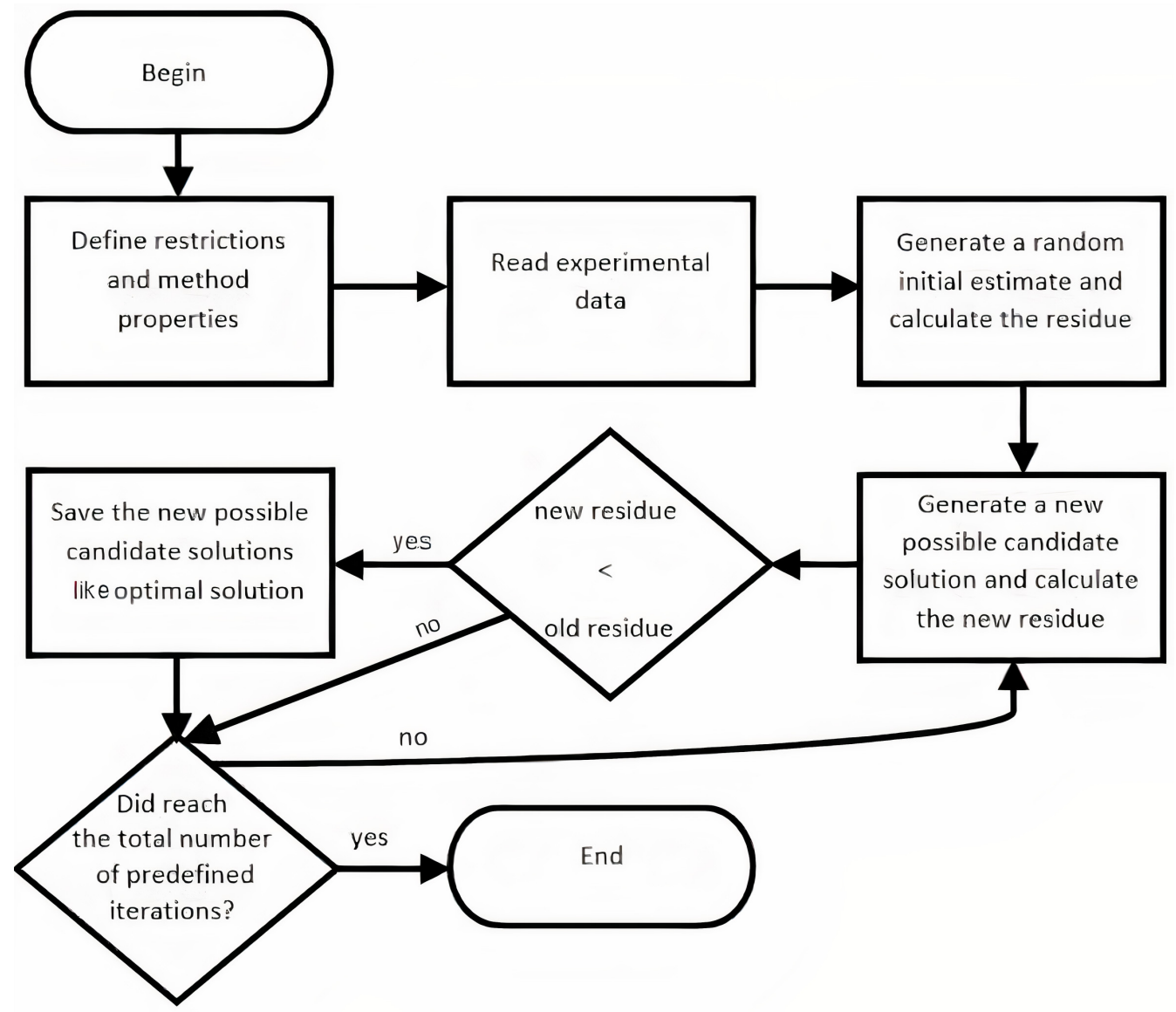

Figure 5: Flowchart of the Luus-Jaakola method.

\subsection{Test case 01 - van Genuchten}

In this case it is considered a depth, $L z=60 \mathrm{~cm}$, time of $1200 \mathrm{~s}$, and conditions of the Dirichlet type. The initial and boundary conditions are described below,

$$
\left\{\begin{array}{l}
\psi(z, 0)=-350.0 \mathrm{~cm}, 0<z<L z \\
\psi(0, t)=-10.0 \mathrm{~cm}, t>0 \\
\psi\left(L_{z}, t\right)=-350.0 \mathrm{~cm}, t>0
\end{array}\right.
$$

Consider the values of the parameters of the problem, $K_{s}=6.2611 \cdot 10^{-3} \mathrm{~cm} \mathrm{~s}^{-1}, \alpha=2.80$. $10^{-2} \mathrm{~cm}, n=2.239, m=0.5534, \theta_{r}=0.029 \mathrm{~cm}^{3} \mathrm{~cm}^{-3}$ and $\theta_{s}=0.366 \mathrm{~cm}^{3} \mathrm{~cm}^{-3}$. The experimental data are synthetic. They were obtained solving the direct problem with these parameters to minimize $Q(\mathbf{P})$.

The values of the parameters obtained with the iterative procedure for the solution of the inverse problem are presented in Table 1. Statistical data that measures the accuracy and precision of the 
model are $r^{2}=0.991$ and $d=0.998$, [21], very close to the ideal value 1 , and the residue, eq. 2.2, was $1.95 \cdot 10^{-3}$. In Figure 6 a it is shown the calculated values for the soil moisture content after the solution of the inverse problem, i.e., using the parameters obtained by the Luus-Jaakola method for the van Genuchten retention curve.

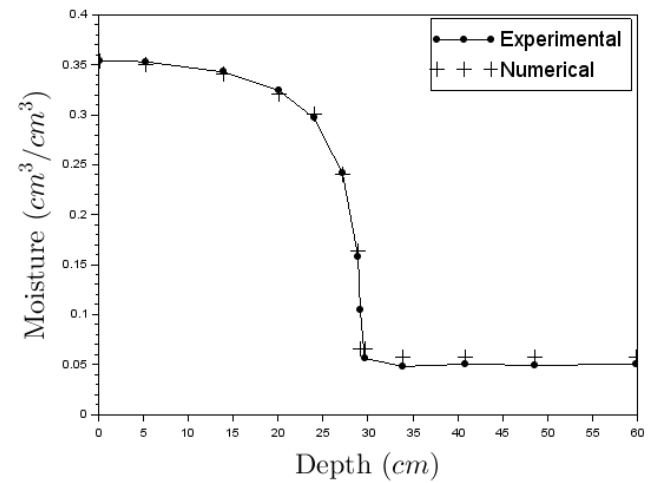

(a) van Genuchten

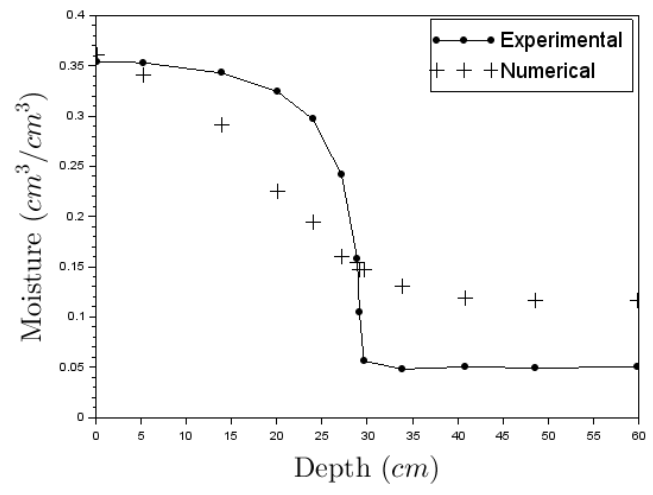

(b) Haverkamp

Figure 6: Solution for the soil moisture content considering van Genuchten's retention curve (a) with $N_{\text {out }}=150, N_{\text {int }}=50$ and $\varepsilon=0.2$, and Haverkamp retention curve (b) with $N_{\text {out }}=200$, $N_{\text {int }}=25$ and $\varepsilon=0.2$, in the Luus-Jaakola method.

Table 1: Parameters of the van Genuchten's and Haverkamp's retention curve.

\begin{tabular}{|c|c|c|c|c|c|c|c|}
\hline & & $\alpha\left(\mathrm{cm}^{-1}\right)$ & $n$ & $m$ & $\theta_{r}\left(\mathrm{~cm}^{3} \mathrm{~cm}^{-3}\right)$ & $\theta_{s}\left(\mathrm{~cm}^{3} \mathrm{~cm}^{-3}\right)$ & $K_{s}\left(c m s^{-1}\right)$ \\
\hline VG & & $2.80 \cdot 10^{-2}$ & 2.239 & 0.5534 & 0.029 & 0.366 & $6.2611 \cdot 10^{-3}$ \\
\hline LJ & & $2.489 \cdot 10^{-2}$ & 1.936 & 0.483 & 0.012 & 0.365 & $6.2611 \cdot 10^{-3}$ \\
\hline $\mathrm{HK}$ & $B$ & $\lambda(\mathrm{cm})$ & A & $\phi$ & $\theta_{r}\left(\mathrm{~cm}^{3} \mathrm{~cm}^{-3}\right)$ & $\theta_{s}\left(\mathrm{~cm}^{3} \mathrm{~cm}^{-3}\right)$ & $K_{s}\left(c m s^{-1}\right)$ \\
\hline & $2.907 \cdot 10^{6}$ & 4.12 & $3.85 \cdot 10^{6}$ & 3.46 & 0.049 & 0.391 & $5.8450 \cdot 10^{-3}$ \\
\hline
\end{tabular}

Legend: HK - Haverkamp; LJ - Luus-Jaakola; VG - van Genuchten

In the process of obtaining Haverkamp parameters for the same case, led to the fowlling statistical data that measures the accuracy and precision of the model: $r^{2}=0.777$ and $d=0.890$, and the residue was $6.05 \cdot 10^{-2}$.

Figure $6 \mathrm{~b}$ shows the obtained Haverkamp retention curve. The adjusted parameters do not represent the experimental data well. This may be linked to the results obtained from the sensitivity coefficients obtained from the Haverkamp retention curve parameters presented in the section 3.2 , where it reports the possible difficulty in estimating some parameters simultaneously. 


\subsection{Test case 02 - Haverkamp}

In this case it is considered a depth, $L z=100 \mathrm{~cm}$, time of $360 \mathrm{~s}$, and initial and boundary conditions of the Dirichlet type. The conditions are described below.

$$
\left\{\begin{array}{l}
\psi(z, 0)=-61.50 \mathrm{~cm}, 0<z<L z \\
\psi(0, t)=-20.73 \mathrm{~cm}, t>0 \\
\psi\left(L_{z}, t\right)=-61.50 \mathrm{~cm}, t>0
\end{array}\right.
$$

Consider the values of the parameters of the problem, $K_{s}=9.44 \cdot 10^{-3}\left(\mathrm{~cm} \mathrm{~s}^{-1}\right), A=1.19 \cdot 10^{6}$, $\phi=4.74, B=1.611 \cdot 10^{6}, \lambda=3.96(\mathrm{~cm}), \theta_{r}=0.075\left(\mathrm{~cm}^{3} \mathrm{~cm}^{-3}\right)$ and $\theta_{s}=0.287\left(\mathrm{~cm}^{3} \mathrm{~cm}^{-3}\right)$. The experimental data are synthetic. They were obtained solving the direct problem with these parameters to minimize $Q(\mathbf{P})$.

The inverse problem was solved to obtain four parameters: $A, B, \lambda$ and $\phi$. Simulations were performed with various values for $N_{\text {out }}$ and $N_{\text {int }}$. The best configuration was for $N_{\text {out }}=200$ and $N_{\text {int }}=25$. Figure 7 shows the soil moisture content based on the parameters obtained by the Luus-Jaakola method, see Table 2, for Haverkamp's retention curve. Good results were obtained, whose statistical indices are $r^{2}=0.995$ and $d=0.990$, [21].

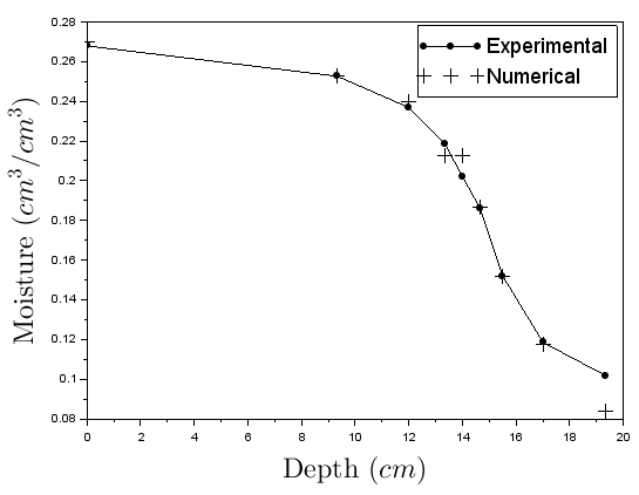

(a) Haverkamp

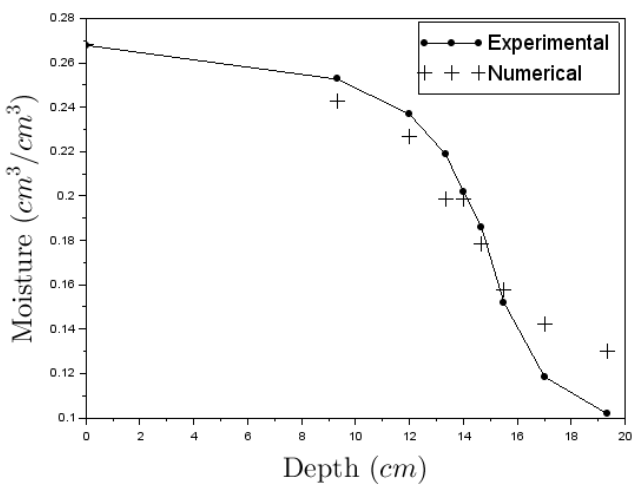

(b) van Genuchten

Figure 7: Solution for the soil moisture content considering Haverkamp's retention curve (a) with $N_{\text {out }}=200, N_{\text {int }}=25$ and $\varepsilon=0.2$, and van Genuchten's retention curve (a) with $N_{\text {out }}=150$, $N_{\text {int }}=50$ and $\varepsilon=0.2$, in the Luus-Jaakola Method.

In the process of obtaining the van Genuchten parameters for the same case, which was obtained using the Haverkamp retention curve in the literature, led to the following statistical data that measures the accuracy and precision of the model: $r^{2}=0.960$ and $d=0.980$, and the residue was $2.03 \cdot 10^{-3}$. 
Table 2: Parameters of the Haverkamp's and van Genuchten's retention curve.

\begin{tabular}{lccccccc}
\hline & $B$ & $\lambda(\mathrm{cm})$ & $A$ & $\phi$ & $\theta_{r}\left(\mathrm{~cm}^{3} \mathrm{~cm}^{-3}\right)$ & $\theta_{s}\left(\mathrm{~cm}^{3} \mathrm{~cm}^{-3}\right)$ & $K_{s}\left(\mathrm{cms}^{-1}\right)$ \\
\hline HK & $1.611 \cdot 10^{6}$ & 3.96 & $1.19 \cdot 10^{6}$ & 4.74 & 0.075 & 0.287 & $9.44 \cdot 10^{-3}$ \\
LJ & $1.130 \cdot 10^{6}$ & 3.87 & $1.862 \cdot 10^{6}$ & 4.82 & 0.053 & 0.294 & $9.87 \cdot 10^{-3}$ \\
\hline VG & & $\alpha\left(\mathrm{cm}^{-1}\right)$ & $n$ & $m$ & $\theta_{r}\left(\mathrm{~cm}^{3} \mathrm{~cm}^{-3}\right)$ & $\theta_{s}\left(\mathrm{~cm}^{3} \mathrm{~cm}^{-3}\right)$ & $K_{s}\left(\mathrm{cms}^{-1}\right)$ \\
\hline & & $2.24 \cdot 10^{-2}$ & 3.00 & 0.6667 & 0.016 & 0.285 & $9.500 \cdot 10^{-3}$ \\
\hline
\end{tabular}

Legend: HK - Haverkamp; LJ - Luus-Jaakola; VG - van Genuchten

\section{CONCLUSIONS}

The Luus-Jaakola's method yielded good results, as can be observed in the statistical data presented, as well as in the moisture content profiles for the estimation of the van Genuchten and Haverkamp retention curve parameters. Being the Luus-Jaakola a probabilistic method, there is always the possibility of not returning the expected values, as happened with test cases with small values for $N_{\text {out }}$ and $N_{\text {int }}$. As a future work it will be considered the implementation of a modified Luus-Jaakola's method, see ref. [10], in order to compare the accuracy of the method and reduce the computational time.

\section{Acknowledgements}

The authors acknowledge the financial support provided by FAPERJ, CNPq and CAPES (finance code 001), as well as the graduate programs in Computational Modeling (IPRJ/UERJ) for the opportunity, in Biosystems Engineering (UFF) for the support, and Computational Modeling in Science and Technology (UFF), and finally, to the Department of Nature Sciences of the UFF for its support to the Postdoctoral Researcher.

\section{REFERENCES}

[1] SciLab. URL https://www.scilab.org. Accessed: 2016-01-15.

[2] V. Baron, Y. Coudière \& P. Sochala. Comparison of DDFV and DG methods for ow in anisotropic heterogeneous porous media. Gas Science and Technology - Revue dÍFP Energies nouvelles, 69 (2014), 673-686.

[3] G.S.A. Campbell. Simple method for determining unsaturated conductivity from moisture retention data. Soil Science, 117:6 (1974), 311-314.

[4] M.A. Celia \& E.T. Bouloutas. A general mass-conservative numerical solution for unsaturated fow equation. Water Resources Rechearch, 26:7 (1990), 1483-1496.

[5] M.X. Guterres. "Evaluation of the Picard-Krylov and Newton-Krylov algorithms in the solution of the Richards equation (in Portuguese)". Ph.D. thesis, IPRJ, Universidade do Estado do Rio de Janeiro, Nova Friburgo, RJ (2014). 
[6] M.X. Guterres, J.F.V. Vasconcellos, A.J. Silva-Neto, C.B.L. Peralta \& L.G.M.F. Teixeira. Approximation of a Solution for the Richards 2D Equation by the Finite Volume Method with the Aid of the Picard-Krylov Algorithms (In Portuguese). Águas Subterrâneas, 31:2 (2017), 89-108.

[7] D.M. Hamby. A review of techniques for parameter sensitivity analysis of environmental models. Environmental Monitoring and Assessment, 32 (1994), 135-154.

[8] R. Haverkamp, M. Vauclin, J. Touma, P.J. Wierenga \& G. Vachaud. A comparison of numerical simulation models for one-dimensional infiltration. Soil Science Society of America Journal, 41:2 (1977), 285-294.

[9] M. Hayek. Analytical solution to transient Richardséquation with realistic water profiles for vertical infiltration and parameter estimation. Water Resources Research, 52:6 (2016), 4438-4457.

[10] J. Jezóowski, R. Bochenek \& G. Ziomek. Random Search Optimization Approach for Highly MultiModal Nonlinear Problems. Advances in Engineering Software, 36 (2005), 504-517.

[11] J.G. Kroes, J.C. van Dam, P. Groenendijk, R.F.A. Hendriks \& C.M.J. Jacobs. "SWAP version 3.2. Theory description and user manual”. ISSN 1566-7197. Wageningen, Alterra, Alterra Report 1649(02) (2008).

[12] R. Luus \& T.H.I. Jaakola. Optimization by Direct Search and Systematic Reduction of the Size of Search Region. AIChE Journal, 19:4 (1973), 760-766.

[13] F.D. Moura-Neto \& A.J. Silva-Neto. "An Introduction to Inverse Problems with Applications". Springer-Verlag (2013).

[14] L.A. Richards. Capillary conduction of liquids through porous mediums. Journal of Applied Physics, 1 (1931), 318. doi:10.1063/1.1745010.

[15] W.O. Sawadogo, P.O.F. Ouedraogo, O. So, G. Barro \& B. Some. Identification of Parameters of Richards Equation Using Modified Hybrid Grey Wolf Optimizer-Genetic Algorithm (HmGWOGA). European Journal of Pure and Applied Mathematics, 12:4 (2019), 1567-1583.

[16] A.J. Silva-Neto, J.C. Becceneri \& H.F.C. Velho. "Computational Intelligence Applied to Inverse in Radiative Transfer Problems (In Portuguese)”. EdUERJ, Rio de Janeiro (2016).

[17] A.J. Silva-Neto \& R.E. White. Numerical Solution of Fluid Flow in Partially Saturated Porous Media. Transactions of the Eleventh Army Conference on Applied Mathematics and Computing, ARO Report 94-1, Carnegie Mellon University, 2:2 (1994), 353-371.

[18] M.O. Temperini. "Inverse modeling to obtain parameters for Richards equation (in Portuguese)". Ph.D. thesis, PGEB, Universidade Federal Fluminense, Niterói, RJ (2018).

[19] M.T. van Genuchten. A closed-form equation for predicting the hydraulic conductivity of unsaturated soils. Soil Science Society of America Journal, 44:5 (1980), 892-898.

[20] M. Vocciante, A.P. Reverberi \& V.G. Dovì. Generalisation of the Solution of the Inverse RichardsṔroblem. Chemical Engineering Transactions, 52 (2016), 1285-1290.

[21] C.J. Willmott. On the validation of models. Physical Geography, 2:2 (2013), 1-7. 
[22] Y. Zha, J. Yang, J. Zeng, C.H.M. Tso, W. Zeng \& L. Shi. Review of numerical solution of RichardsonRichards equation for variably saturated flow in soils. Wiley Interdisciplinary Reviews: Water, g:2 (2019), e1364.

(cc) BY 